\title{
Cdk5 is essential for adult hippocampal neurogenesis
}

\author{
Diane C. Lagace ${ }^{a}$, David R. Benavidesa, Janice W. Kansya, Marina Mapellib, Paul Greengardc,1, James A. Bibba, \\ and Amelia J. Eischa,1 \\ aDepartment of Psychiatry, University of Texas Southwestern Medical Center, 5323 Harry Hines Boulevard, Dallas, TX 75390-9070; bStructural Biology Unit, \\ Department of Experimental Oncology, European Institute of Oncology, via Adamello 16, 20139 Milan, Italy; and 'Laboratory of Molecular and Cellular \\ Neuroscience, The Rockefeller University, 1230 York Avenue, New York, NY 10021
}

Contributed by Paul Greengard, October 9, 2008 (sent for review October 1, 2008)

The molecular factors regulating adult neurogenesis must be understood to harness the therapeutic potential of neuronal stem cells. Although cyclin-dependent kinase 5 (Cdk5) plays a critical role in embryonic corticogenesis, its function in adult neurogenesis is unknown. Here, we assessed the role of Cdk5 in the generation of dentate gyrus (DG) granule cell neurons in adult mice. Cre recombinase-mediated conditional knockout (KO) of Cdk5 from stem cells and their progeny in the DG subgranular zone (SGZ) prevented maturation of new neurons. In addition, selective KO of Cdk5 from mature neurons throughout the hippocampus reduced the number of immature neurons. Furthermore, Cdk5 gene deletion specifically from DG granule neurons via viral-mediated gene transfer also resulted in fewer immature neurons. In each case, the total number of proliferating cells was unaffected, indicating that Cdk5 is necessary for progression of adult-generated neurons to maturity. This role for Cdk5 in neurogenesis was activating-cofactor specific, as p35 KO but not p39 KO mice also had fewer immature neurons. Thus, Cdk5 has an essential role in the survival, but not proliferation, of adult-generated hippocampal neurons through both cell-intrinsic and cell-extrinsic mechanisms.

cyclin-dependent kinase | dentate gyrus granule cell | doublecortin | nestin-CreERT2 | viral-mediated gene transfer

$\mathrm{n}$ the adult brain, neural precursors give rise to immature neurons which mature into granule cells in the hippocampal subgranular zone (SGZ) $(1,2)$. This process of adult neurogenesis is orchestrated by signaling intrinsic to the new cells as well as extrinsic or microenvironmental influences in the SGZ niche $(2,3)$. For example, the orphan nuclear receptor tailless intrinsically regulates stem cell proliferation (4) and neurogenesin-1 extrinsically regulates fate specification (5). In contrast to our growing understanding of factors that regulate proliferating cells, the identity and mechanisms of factors that regulate immature neurons remain unclear (3). Elucidation of the intrinsic and extrinsic factors that mediate the formation of new neurons is critical to understand normal brain development and to realize the therapeutic potential of adult neurogenesis.

Cyclin-dependent kinase $5(\mathrm{Cdk} 5)$ is a proline-directed, serine/threonine cyclin-dependent kinase family member. Its activity is largely restricted to post-mitotic neurons of the central nervous system (6) and depends on association with the noncyclin cofactors p35 and p39 $(7,8)$. Cdk5 plays a critical role in neuronal migration during development and is highly enriched in the hippocampus. Therefore, it might be predicted to be important in the formation of immature neurons in the adult hippocampus. This hypothesis is supported by the importance of Cdk5 substrates, including nestin and doublecortin (DCX), in adult neurogenesis $(9-11)$. Here, we demonstrate that Cdk5 is critical for adult hippocampal neurogenesis. Using several approaches, we show that ablation of the Cdk5 gene from either SGZ stem cells or from mature dentate gyrus (DG) neurons decreases the number of immature SGZ neurons. These findings emphasize that Cdk5 regulates neurogenesis through both intrinsic and extrinsic mechanisms in the SGZ of the adult hippocampus.
Results

Cdk5 Protein Is Located in Immature Neurons and Mature Granule Neurons of the DG. Previous in situ hybridization and immunoblot analyses reported high levels of Cdk5 in the embryonic and adult DG (12-14). To identify which DG cells specifically express Cdk5 in the adult, a Cdk5 monoclonal antibody (Ab) was generated. Validation of the Ab demonstrated its specificity in vitro and in vivo (see supporting information (SI) Fig. S1) and localization of Cdk5 in adult hippocampal cells (Fig. 1A). Interestingly, Cdk5 was not present in dividing stem-like and progenitor cells, as demonstrated by the lack of colabeling of Cdk5 and green fluorescent protein (GFP) in the SGZ of nestin-GFP mice (Fig. $1 B$ ). In contrast, Cdk5 staining was evident in a subpopulation $(\approx 10 \%)$ of "older" post-mitotic immature DCX immunoreactive (DCX+) neurons (11), identified by their immature apical branching dendrites (15) (Fig. 1C). As expected, Cdk5 was also expressed in almost all (approximately $>95 \%$ ) mature granule neurons $(\mathrm{NeuN}+$; Fig. $1 D)$. Thus, $\mathrm{Cdk} 5$ is not expressed at early stages of adult neurogenesis, but in later stages, with most mature granule cell neurons expressing Cdk5.

Inducible Removal of Cdk5 from SGZ Stem Cells Prevents Adult Hippocampal Neurogenesis. Cdk5 $5^{-1-} \mathrm{KO}$ mice are perinatal lethal (16), impeding examination of the role of Cdk5 in adult hippocampal neurogenesis. Therefore, we created an inducible nestin driven Cdk5 KO transgenic mouse (Cdk5 nKO) that allows specific ablation of Cdk5 from nestin expressing progenitor cells and their progeny. Nestin-CreER 2 /R26R-YFP (17) mice were crossed with floxed $\mathrm{Cdk} 5$ (fCdk5) mice $(18,19)$ to generate Cdk5 nKO mice (Fig. $2 A$ ). In these mice, the nestin promotor drives the expression of the Cre recombinase-modified estrogen receptor $\left(\mathrm{CreER}^{\mathrm{T} 2}\right)$ fusion protein in SGZ progenitors. On administration of the estrogen congener tamoxifen (TAM), CreER $^{\mathrm{T} 2}$ recombines DNA loxP sites, allowing YFP expression and Cdk5 deletion in nestin expressing cells (Fig. $2 A$ ). In mice harboring the WT Cdk5 gene, the number of recombined $\left(\mathrm{YFP}^{+}\right)$SGZ cells doubled between 12 and 30 days post-TAM (Fig. $2 B$ and $C$ ) because of the accumulation of recombined dividing progenitor progeny (17). In contrast, there was no change in YFP+ SGZ cells in Cdk5 $\mathrm{nKO}$ mice between 12 and 30 days post-TAM (Fig. $2 B$ and $C$ ). Thus, removal of Cdk5 from stem cells and their progeny prevents the increase in the number of recombined cells 30 days post-TAM.

We hypothesized that the removal of $\mathrm{Cdk} 5$ reduced the number of YFP + cells by preventing the maturation of DCX+

Author contributions: D.C.L., D.R.B., P.G., J.A.B., and A.J.E. designed research; D.C.L., D.R.B., J.W.K., M.M., and A.J.E. performed research; J.W.K., M.M., P.G., J.A.B., and A.J.E. contributed new reagents/analytic tools; D.C.L. and A.J.E. analyzed data; and D.C.L., D.R.B., P.G. J.A.B., and A.J.E. wrote the paper

The authors declare no conflict of interest.

${ }^{1}$ To whom correspondence may be addressed. E-mail: amelia.eisch@utsouthwestern.edu or greengard@mail.rockefeller.edu.

This article contains supporting information online at www.pnas.org/cgi/content/full/ 0810137105/DCSupplemental.

C 2008 by The National Academy of Sciences of the USA 

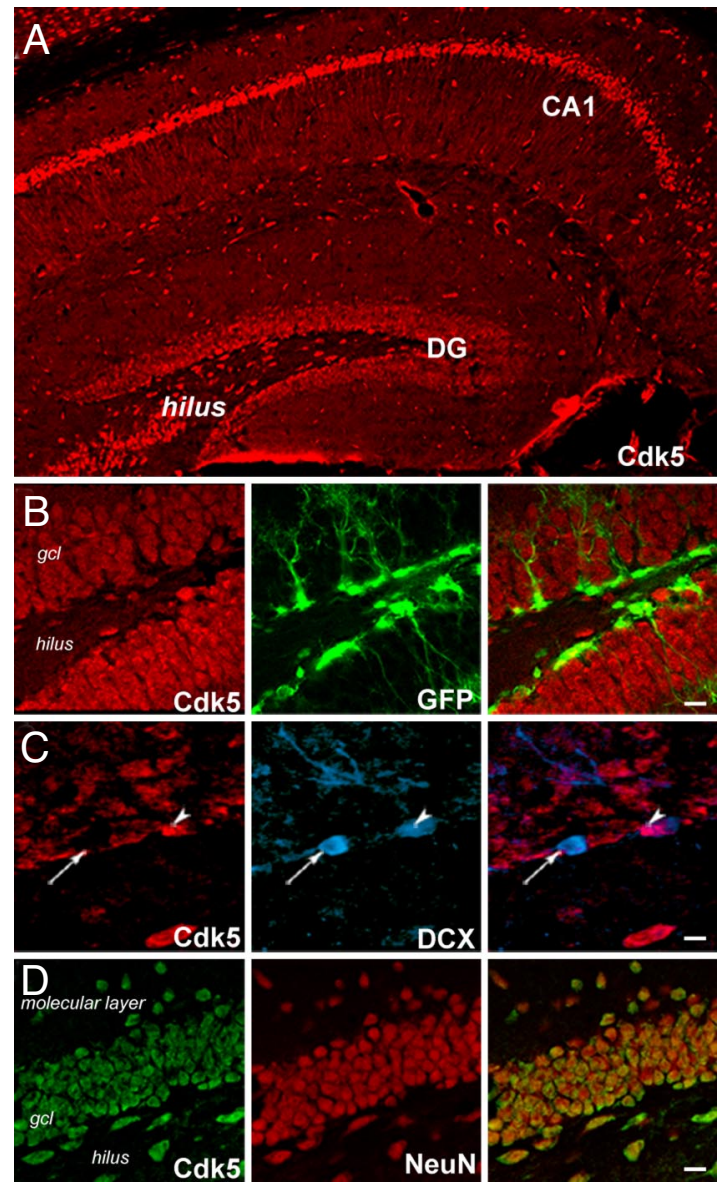

Fig. 1. Localization of $\mathrm{Cdk} 5$ in postmitotic immature and mature neurons in the hippocampus. (A) Immunofluorescent detection of $\mathrm{Cdk} 5$ in dentate gyrus (DG) granule cells, interneurons of the hilus, and dendrites and cell bodies of CA1-CA3 neurons. $(B)$ Cdk5 and GFP immunolabeling showing that GFP+ progenitors in SGZ of nestin-GFP reporter mouse do not express Cdk5. (C) Cdk5 and DCX immunolabeling of SGZ showing Cdk5 in a subpopulation of DCX+ immature neurons (arrowhead). An arrow indicates a typical DCX+ cell that does not express Cdk5. (D) Cdk5 and NeuN immunolabeling illustrating that almost all DG granule cells (NeuN+) also express Cdk5. (Scale bars: $B, 60 \mu \mathrm{m}$; C, $30 \mu \mathrm{m} ; D, 60 \mu \mathrm{m}$.)

cells into neurons because $(i) \mathrm{WT}$ and $\mathrm{Cdk} 5 \mathrm{nKO}$ mice did not differ in the number of YFP + progenitor cells 12 days postTAM (Fig. 2C); (ii) Cdk5 is expressed in DCX + and mature $\mathrm{NeuN}+\mathrm{SGZ}$ cells (Fig. $1 C$ and $D$ ); and (iii) $\mathrm{YFP}+/ \mathrm{NeuN}+$ neurons first appear 30 days post-TAM (17). Indeed WT mice had mature $(\mathrm{NeuN}+) \mathrm{YFP}+$ neurons 30 days post-TAM, but Cdk5 nKO mice had $<1.5 \%$ YFP + cells expressing NeuN (Fig. $2 D)$. This occurred without a change in the percentage of YFP+ cells that expressed DCX $(\mathrm{WT}=36.3 \% \pm 4.7 ; \mathrm{nKO}=$ $32.1 \% \pm 7.3)$. Together these findings suggest that the reduction in number of mature neurons 30 days post-TAM in Cdk5 $\mathrm{nKO}$ mice may be a result of enhanced cell death in the SGZ. In fact, there were significantly more activated caspase 3 immunoreactive $(\mathrm{AC} 3+)$ SGZ cells at 30 days post-TAM in Cdk5 nKO mice vs. WT controls (Fig. 2E). Furthermore, embryonic conditional Cdk5 KO mice have no difference in subventricular zone proliferation when examined at an early postnatal time point (20). Thus, these data suggest a cell intrinsic effect, where Cdk5 expression in immature neurons is required for survival of adult-generated dentate granule neurons.

Conditional CamKII-Cdk5 KO Mice and p35 KO Mice Have Fewer Immature SGZ Neurons. Considering the high level of expression of $\mathrm{Cdk} 5$ in mature granule cells and their influence on neurogenesis
A

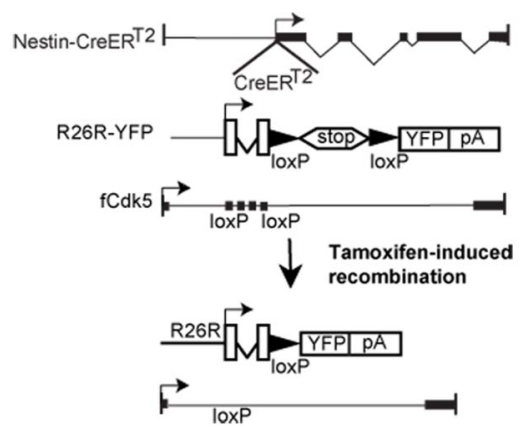

B
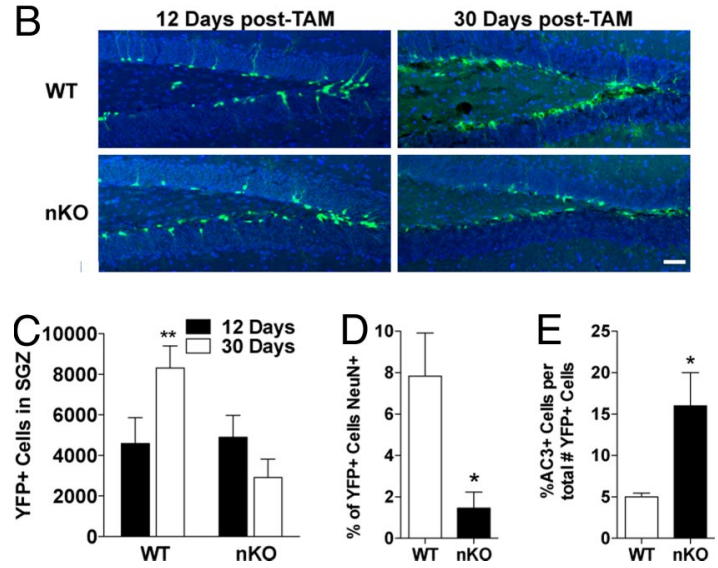

Fig. 2. Ablation of $\mathrm{Cdk} 5$ in nKO mice prevents formation of new neurons. $(A)$ Diagram of genetic manipulations in the 3 mouse lines to create Cdk5 nKO mice. (B) Immunofluorescent detection of YFP+ (green) cells with nuclear counterstaining (DAPI) in the SGZ of WT and Cdk5 nKO mice at 12 and $30 \mathrm{~d}$ post-TAM with quantification $(C: * *, P<0.01$ compared with $12 \mathrm{~d}$ WT, two-way ANOVA; $n=5-10$ per group). (Scale bar, $60 \mu \mathrm{m}$.) (D) Percent YFP+ cells that are mature neurons $(\mathrm{NeuN}+) 30 \mathrm{~d}$ post-TAM $\left(\mathrm{t}_{(4)}=2.9, P<0.05\right) ; n=$ 4 per group). (E) Percent AC3 + per total YFP+ cells at $30 \mathrm{~d}$ post-TAM $\left(\mathrm{t}_{(8)}=2.2\right.$ $P<0.05 ; n=6$ per group).

$(21,22)$, we next deleted Cdk5 from mature granule neurons and evaluated proliferation and maturation. For this study CamKIICre fCdk5 conditional knockout mice (cKO) were used (19). Cdk5 cKO mice had less Cdk5 in the hippocampus when compared with WT mice (WT $=100 \pm 21.6 \%, \mathrm{cKO}=37.8 \pm$ $16.2 \% ; \mathrm{t}_{(10)}=2.4, P<0.05 ; n=6$ per group).

The fCdk $5 \mathrm{cKO}$ mice exhibited no difference in the number of proliferating cells $(\mathrm{Ki} 67+)$ compared with WT mice (Fig. $3 A$ ). However, there was a $42 \%$ reduction in DCX + cells (Fig. $3 B$ and $C)$. To confirm that recombination in the cKO mice only occurred in mature granule cells, we bred cKO mice with R26R-YFP reporter mice. A fraction of mature NeuN + granule cells $(39.9 \pm 1.8 \%)$ were YFP+ (Fig. $3 E)$. Almost all YFP + cells were NeuN+, but no YFP + cells were DCX + (Fig. 3D). These results indicate that in addition to its intrinsic influence, $\mathrm{Cdk} 5$ plays an extrinsic role in the maturation of adult-generated SGZ neurons.

Mice lacking the Cdk5 activating cofactor p35 are viable (23) allowing for the comparison of adult neurogenesis in p35 KO and our conditional KO mice. WT and p35 KO mice had similar numbers of proliferating SGZ cells (Ki67+; Fig. $3 F$ ) but, interestingly, there was a $30 \pm 7 \%$ reduction in the number of immature $(\mathrm{DCX}+)$ SGZ neurons in $\mathrm{p} 35 \mathrm{KO}$ mice compared with WT mice (Fig. $3 G$ and $H$ ). In addition to p35, its homolog p39 also activates Cdk5 and is present in comparable levels in adult brain (12). However, analysis of p39 $\mathrm{KO}$ mice revealed no significant neurogenic phenotype (data not shown). These data confirm the importance of $\mathrm{Cdk} 5$ in the development of immature 

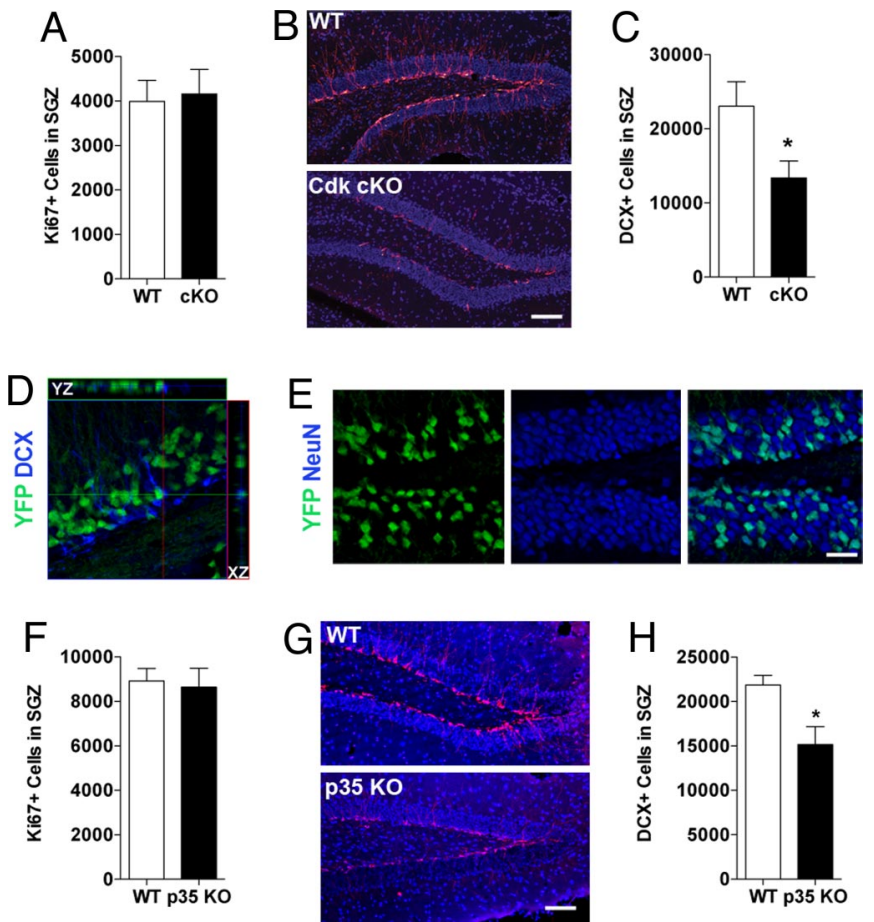

Fig. 3. Ablation of $\mathrm{Cdk} 5 \mathrm{in} \mathrm{Cdk} 5 \mathrm{cKO}$ mice reduces the number of immature neurons. (A) Number of proliferating cells (Ki67+) in WT and Cdk5 cKO mice. Representative images $(B)$ and quantification $(C)$ of immature neurons in DG of WT vs. Cdk5 cKO mice via DCX + cell counts $\left(t_{(9)}=2.2, P<0.05 ; n=4\right.$ per group; DCX, red; DAPI blue). (D) Orthogonal image of immunolabeling for $\mathrm{DCX}+$ and Cre reporter YFP + cells. Note DCX + cells exhibit no Cre activity (YFP+) in Cdk5 cKO mice. (E) YFP and NeuN immunolabeling illustrating YFP+ cells are mature neurons (NeuN + ) in $\mathrm{Cdk} 5 \mathrm{cKO}$ mice. (F) Number of dividing $(\mathrm{Ki67}+)$ cells in WT and p35 KO mice. (G) Immunolabeling of DCX+ immature neurons in DG of WT and p35 KO mice with quantification $\left(H, \mathrm{t}_{(6)}=2.9, P<\right.$ $0.05 ; n=4$ per group). (Scale bars: $B$ and $G, 100 \mu \mathrm{m} ; E, 60 \mu \mathrm{m}$.)

neurons and indicate that the $\mathrm{Cdk} 5 / \mathrm{p} 35$ complex predominantly regulates adult SGZ neurogenesis.

Selective Deletion of Cdk5 in Mature DG Neurons Attenuates Neurogenesis. To further investigate the extrinsic role of $\mathrm{Cdk} 5$ in hippocampal neurogenesis, $\mathrm{Cdk} 5$ was specifically removed from postmitotic DG neurons via adeno-associated virus (AAV)mediated delivery of Cre recombinase. AAV-Cre was stereotaxi- cally infused unilaterally into the DG of R26R-YFP/fCdk5 mice and histological analysis was conducted. Recombination, as assessed by YFP expression 2 weeks after infusion, was evident in anterior DG granule neurons and hilar interneurons (Fig. $4 \mathrm{~A}$ and $B$ ). Viral-mediated transduction of the DG of R26R-YFP/ fCdk5 but not WT mice decreased the number of immature $(\mathrm{DCX}+)$ neurons in the injected side by $21 \%$ compared with the contralateral side (Fig. $4 C-E$ ). As in our other models, proliferation was the same between WT and fCdk5 mice (Fig. 4E). These data underscore that ablation of Cdk5 from mature neurons in the DG impedes the development of new SGZ neurons in the absence of altering SGZ proliferation.

\section{Discussion}

Adult hippocampal neurogenesis is a dynamic process comprised of the birth, migration, differentiation, survival, and functional integration of new neurons in the granule cell layer (3). Within a week of cell cycle exit, new hippocampal progenitors extend dendrites toward the molecular layer, and within two weeks extend their mossy fiber axons through the hilus to CA3 (3). Within four weeks approximately half of the new cells in the postnatal hippocampus have survived to integrate into the granule cell layer circuitry, a process reminiscent of pruning during embryogenesis (24). Progression through each stage is achieved via intrinsic alterations within the differentiating and migrating progenitors as well by a host of extrinsic factors in the hippocampal niche (3). While neurogenesis research has the potential for translation to clinical therapies aimed at cell replacement for neurodegenerative disorders, understanding the factors that govern cell survival and neuronal integration is critical.

Cdk5 regulates actin dynamics, microtubule stability, cell adhesion, axon guidance, and membrane transport through the phosphorylation of a large number of substrates $(25,26)$. Cdk5 was first implicated in embryonic cortical migration and has recently been identified as a regulator of postnatal subventricular zone neuroblast migration $(16,20,27)$. Here, we identify $\mathrm{Cdk} 5$ as a novel factor that acts both intrinsically and extrinsically to regulate the survival of adult-generated hippocampal neurons. The migration of new neurons in the hippocampus was not overtly modified in any of our Cdk5 KO mouse models, which could be attributed to the requirement of Cdk5 for survival. Our data demonstrate that hippocampal Cdk5 is essential for survival of adult-generated neurons, but not for the survival of preexisting mature granule neurons or hilar interneurons. Whereas the survival of cells in vitro may be Cdk5-dependent (28-30), the
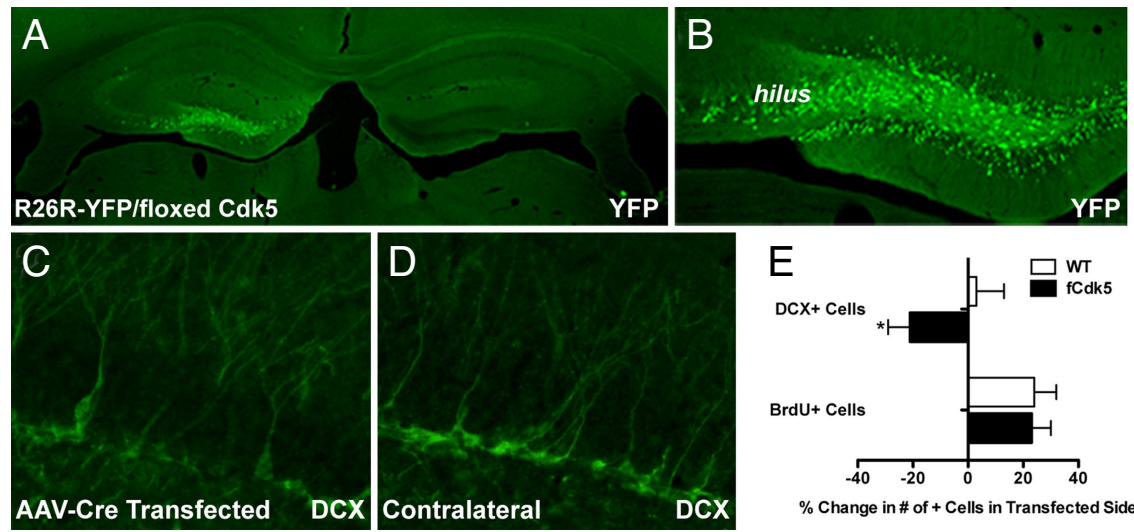

Fig. 4. Viral-mediated ablation of $\mathrm{Cdk} 5$ from mature granule cells reduces immature neuron number. Immunolabeling of YFP+ cells in YFP reporter fCdk5 hippocampus $14 \mathrm{~d}$ after unilateral AAV-Cre transduction (left) at low $(A)$ and high $(B)$ magnifications. $(C$ and $D)$ Immunofluorescent staining of immature (DCX + ) neurons in AAV-Cre transduced DG $(C)$ and contralateral hemisphere $(D)$. (E) Quantification of $D C X+$ and $B r d U+$ cells $\left(\mathrm{t}_{(8)}=2.2, P<0.05 ; n=5\right.$ per group). 
present study shows that the in vivo regulation of neuronal survival by Cdk5 is cell-type specific.

Proliferation and survival of progenitor neurons are likely important contributors to the composition and function of the adult hippocampus. Cdk5 appears to predominantly function in the regulation of pathways involved in the survival of these neurons. Indeed numerous intrinsic and extrinsic progenitor survival factors are Cdk5 substrates. For example, DCX and proto-oncogene B-cell lymphoma protein-2 (Bcl-2) are Cdk5 substrates expressed in immature and mature granule neurons that might intrinsically mediate neuronal survival $(10,31,32)$. In parallel, altered $\mathrm{N}$-methyl-D-aspartate glutamate receptor (NMDAR) activity in mature granule neurons may extrinsically mediate neuronal survival $(21,22)$ and $\mathrm{Cdk} 5$ regulates NMDAR-mediated excitatory postsynaptic currents (18).

The present study shows Cdk5 is essential for adult neurogenesis. In adults, both neurogenesis $(33)$ and $\operatorname{Cdk} 5(18,34,35)$ are important in learning and memory. It is also interesting to note that dysregulation of $\mathrm{Cdk} 5$ is implicated in neurodegenerative disorders, such as Alzheimer's disease $(25,26,36)$, which are accompanied by deficiencies in neurogenesis $(25,26,36)$. Thus, it is possible that the cognitive impairments that are hallmarks of these neurodegenerative diseases may involve both the loss of existing neurons associated with aberrant Cdk5 activity and the deleterious effects that Cdk5 dysregulation may impart on hippocampal neurogenesis.

\section{Materials and Methods}

Animals. Mice were housed in an Association for Assessment and Accreditation of Laboratory Animal Care International (AAALAC)-approved facility at UT Southwestern on a 12-h light/dark cycle. The p35 KO (23), p39 KO (37), nestin-GFP reporter (38), fCdk5 (18, 19), CamKII-Cre(T50)-fCdk5 (31), and nestin-CreER ${ }^{\mathrm{T}} / \mathrm{R} 26 \mathrm{R}$-YFP (17) mice have been previously described. Nestin-CreER ${ }^{\mathrm{T}} / \mathrm{R} 26 \mathrm{R}-\mathrm{YFP}$ mice were crossed with $\mathrm{fCdk} 5$ mice to generate Cdk5 nKO mice, which at $5-7$ weeks of age, were given TAM $(180 \mathrm{mg} / \mathrm{kg} /$ day i.p., 5 days) and killed at 12 or 30 days post-TAM. Mice were genotyped as previously published for Cre (39), YFP (40), fCdk5 (18), p35 (23), and p39 (37) alleles.

Dentate-Specific Cdk5 KO via AAV. Serotype 2-rAAV-Cre was generated as previously described (19) and unilaterally delivered to the DG of adult homozygous fCdk5 or WT littermates (10-12 weeks old, coordinates from bregma at skull surface AP-1.7, LM + 1.2, DV-2.4, $1 \mu$ l per injection). Mice received BrdU ( $150 \mathrm{mg} / \mathrm{kg}$, i.p., Roche Molecular Biochemicals) $2 \mathrm{~h}$ before they were killed.

Tissue Collection and Histology. Immunohistochemistry (IHC) was performed as previously described (17). Mice were perfused with $4 \%$ paraformaldehyde and

1. Cameron HA, Woolley CS, McEwen BS, Gould E (1993) Differentiation of newly born neurons and glia in the dentate gyrus of the adult rat. Neuroscience 56:337-344.

2. Kempermann G, Gast D, Kronenberg G, Yamaguchi M, Gage FH (2003) Early determination and long-term persistence of adult-generated new neurons in the hippocampus of mice. Development 130:391-399.

3. Duan X, Kang E, Liu CY, Ming GL, Song H (2008) Development of neural stem cell in the adult brain. Curr Opin Neurobiol 18:108-115.

4. Zhang CL, Zou Y, He W, Gage FH, Evans RM (2008) A role for adult TLX-positive neural stem cells in learning and behavior. Nature 451:1004-1007.

5. Ueki T, et al. (2003) A novel secretory factor, Neurogenesin-1, provides neurogenic environmental cues for neural stem cells in the adult hippocampus. J Neurosci 23:11732-11740.

6. Hellmich MR, Pant HC, Wada E, Battey JF (1992) Neuronal cdc2-like kinase: A cdc2related protein kinase with predominantly neuronal expression. Proc Natl Acad Sci USA 89:10867-10871.

7. Lew J, Beaudette K, Litwin CM, Wang JH (1992) Purification and characterization of a novel proline-directed protein kinase from bovine brain. J Biol Chem 267:13383-13390.

8. Tsai LH, Delalle I, Caviness VS Jr, Chae T, Harlow E (1994) p35 is a neural-specific regulatory subunit of cyclin-dependent kinase 5. Nature 371:419-423.

9. Sahlgren CM, et al. (2003) Cdk5 regulates the organization of Nestin and its association with p35. Mol Cell Biol 23:5090-5106.

10. Tanaka T, Serneo FF, Tseng HC, Kulkarni AB, Tsai LH, Gleeson JG (2004) Cdk5 phosphorylation of doublecortin ser 297 regulates its effect on neuronal migration. Neuron 41:215-227. brains were cryoprotected in 30\% sucrose in $0.1 \mathrm{M} \mathrm{PBS}$, and sectioned (30 $\mu \mathrm{m})$ on a freezing microtome. Slides underwent antigen retrieval $(0.01 \mathrm{M}$ citric acid, $\mathrm{pH} 6.0,100^{\circ} \mathrm{C}$ for $15 \mathrm{~min}$ ) and BrdU-labeled sections underwent membrane permeabilization ( $0.1 \%$ trypsin in $0.1 \mathrm{M}$ Tris and $0.1 \% \mathrm{CaCl}_{2}, 10 \mathrm{~min}$ ), and DNA denaturation ( $2 \mathrm{~N} \mathrm{HCl}$ in $1 \times$ PBS, $30 \mathrm{~min}$ ). To remove endogenous peroxidase activity, sections were incubated with $0.3 \% \mathrm{H}_{2} \mathrm{O}_{2}$ for $30 \mathrm{~min}$. Nonspecific binding was blocked with 3\% donkey serum (Jackson ImmunoLaboratories) and 0.3\% Triton-X in PBS for 30-60 min. Primary Abs included rat anti-BrdU (1:500, Accurate), rabbit AC3 (1:500, Cell Signaling), goat antiDCX (1:3000, Santa Cruz Biotechnology), rabbit anti-GFP (used for GFP and YFP detection; 1:500, Invitrogen), and rabbit anti-Ki67 (1:500, Vector Laboratories). The mouse monoclonal Ab for $\operatorname{Cdk} 5(1: 1,000,1 \mathrm{H3})$ was generated against purified protein at the UT Southwestern Ab Core facility (Fig. S1). For double labeling, primary Abs were simultaneously incubated (AC3/YFP, BrdU/YFP, Cdk5/YFP, DCX/YFP; Ki67/YFP) and processed for each Ab separately. For AC3, BrdU, and NeuN, a fluorescent-tagged secondary Ab (1:200, Jackson ImmunoResearch) was used. For Cdk5, DCX, Ki67 or YFP, a biotin-tagged secondary Ab (1:200, Jackson ImmunoResearch) was followed by ABC (Vector Laboratories) and Tyramide-Plus signal amplification (1:50, PerkinElmer). Slides were counterstained with DAPI (1:5,000, Roche). Immunoblot analysis for Cdk5 was performed as previously described (19).

Quantification of immunoreactive hippocampal cells was performed with an Olympus BX-51 microscope $(400 \times)$ as previously described and validated (41). Briefly, an observer blind to experimental groups counted YFP+ cells via stereology and use of the optical fractionation method through analysis of every 9 th $30-\mu \mathrm{m}$ coronal section throughout the SGZ $(-0.82 \mathrm{~mm}$ to $-4.24 \mathrm{~mm}$ from bregma). For viral-transduced tissue, YFP+ cells were counted in both hemispheres in 3 sections within the injection penumbra with blinding for injection side and genotype. Phenotypic analysis and classification of YFP+ cells $(\approx 50-150$ cells per mouse, $n=4-6$ mice per time point) was performed by using a confocal microscope (Leica TCS SL confocal and Zeiss Axiovert 200 and LSM510-META; emission wavelengths 488, 543, and 633,630× and $1,000 \times)$. For scanning and optical sectioning in the $Z$ plane, image stacks were imported into the 3D Velocity (Improvision) reconstruction program for 3-D rendering.

Statistical Analyses. Data are reported as mean \pm SEM. Experiments with two groups were analyzed by the unpaired $t$ test. Analyses of three or more groups were performed by using ANOVA followed by a Bonferroni post hoc test. Statistical significance was defined as $P<0.05$.

ACKNOWLEDGMENTS. We thank Li-Huei Tsai for use of p35 and p39 KO mice; Masahiro Yamaguchi for the nestin-GFP reporter mice; Pierre Chambon for the Cre-ER ${ }^{\mathrm{T} 2}$ construct; Weimin Zhong for the nestin construct; Andrea Musacchio for providing pure Cdk5 for the generation of antibody; and Eric Nestler for assistance with this study; and Laure Farnbauch, David Pyle, Stephanie Rogan, Greg Wallingford, Sumana Chakravarty, and Ezekiell Mouzon for technical assistance. This work was supported by a postdoctoral fellowship from the Canadian Institute of Health Research (to D.C.L.); National Institutes of Health Grants DA10044 and MH074866 (to P.G.), DA16672 and MH079710 (to J.A.B.), and R01 DA016765, R21 DA023701, and K02 DA023555 (to A.J.E.); and Cure Alzheimer's Fund (P.G.).

11. Rao MS, Shetty AK (2004) Efficacy of doublecortin as a marker to analyze the absolute number and dendritic growth of newly generated neurons in the adult dentate gyrus. Eur J Neurosci 19:234-246.

12. Zheng $M$, Leung $C L$, Liem RK (1998) Region-specific expression of cyclin-dependent kinase 5 (cdk5) and its activators, p35 and p39, in the developing and adult rat central nervous system. J Neurobio/ 35:141-159.

13. Delalle I, Bhide PG, Caviness VS Jr, Tsai LH (1997) Temporal and spatial patterns of expression of p35, a regulatory subunit of cyclin-dependent kinase 5 , in the nervous system of the mouse. J Neurocyto/ 26, 283-296.

14. Wu DC, Yu YP, Lee NT, Yu AC, Wang JH, Han YF (2000) The expression of Cdk5, p35, p39, and Cdk5 kinase activity in developing, adult, and aged rat brains. Neurochem Res 25:923-929.

15. Plumpe T, et al. (2006) Variability of doublecortin-associated dendrite maturation in adult hippocampal neurogenesis is independent of the regulation of precursor cell proliferation. BMC Neurosci 7:77.

16. Ohshima T, et al. (1996) Targeted disruption of the cyclin-dependent kinase 5 gene results in abnormal corticogenesis, neuronal pathology and perinatal death. Proc Natl Acad Sci USA 93:11173-11178.

17. Lagace DC, et al. (2007) Dynamic contribution of nestin-expressing stem cells to adult neurogenesis. J Neurosci 27:12623-12629.

18. Hawasli $A H$, et al. (2007) Cyclin-dependent kinase 5 governs learning and synaptic plasticity via control of NMDAR degradation. Nat Neurosci 10:880-886.

19. Benavides DR, et al. (2007) Cdk5 modulates cocaine reward, motivation, and striatal neuron excitability. J Neurosci 27:12967-12976. 
20. Hirota Y, et al. (2007) Cyclin-dependent kinase 5 is required for control of neuroblast migration in the postnatal subventricular zone. J Neurosci 27:12829-12838.

21. Tashiro A, Sandler VM, Toni N, Zhao C, Gage FH (2006) NMDA-receptor-mediated, cell-specific integration of new neurons in adult dentate gyrus. Nature 442:929933.

22. Ge S, Yang CH, Hsu KS, Ming GL, Song H (2007) A critical period for enhanced synaptic plasticity in newly generated neurons of the adult brain. Neuron 54:559-566.

23. Chae T, Kwon YT, Bronson R, Dikkes P, Li E, Tsai LH (1997) Mice lacking p35, a neuronal specific activator of Cdk5, display cortical lamination defects, seizures, and adult lethality. Neuron 18:29-42.

24. Hayes NL, Nowakowski RS (2002) Dynamics of cell proliferation in the adult dentate gyrus of two inbred strains of mice. Brain Res Dev Brain Res 134:77-85.

25. Dhavan R, Tsai LH (2001) A decade of CDK5. Mol Cell Biol 2:749-759.

26. Cruz JC, Tsai LH (2004) A Jekyll and Hyde kinase: Roles for Cdk5 in brain development and disease. Curr Opin Neurobiol 14:390-394.

27. Hirasawa M, et al. (2004) Perinatal abrogation of Cdk5 expression in brain results in neuronal migration defects. Proc Natl Acad Sci USA 101:6249-6254.

28. Cheung ZH, Ip NY (2004) Cdk5: Mediator of neuronal death and survival. Neurosci Lett 361:47-51.

29. Cheung ZH, Gong K, Ip NY (2008) Cyclin-dependent kinase 5 supports neuronal survival through phosphorylation of Bcl-2. J Neurosci 28:4872-4877.

30. O'Hare MJ, et al. (2005) Differential roles of nuclear and cytoplasmic cyclindependent kinase 5 in apoptotic and excitotoxic neuronal death. $J$ Neurosci 25:8954-8966

31. Kuhn HG, Biebl M, Wilhelm D, Li M, Friedlander RM, Winkler J (2005) Increased generation of granule cells in adult $\mathrm{Bcl}$-2-overexpressing mice: A role for cell death during continued hippocampal neurogenesis. Eur J Neurosci 22:1907-1915.
32. Friocourt G, Liu JS, Antypa M, Rakic S, Walsh CA, Parnavelas JG (2007) Both doublecortin and doublecortin-like kinase play a role in cortical interneuron migration. $J$ Neurosci 27:3875-3883.

33. Zhao C, Deng W, Gage FH (2008) Mechanisms and functional implications of adult neurogenesis. Cell 132:645-660.

34. Fischer A, Sananbenesi F, Schrick C, Spiess J, Radulovic J (2003) Regulation of contextual fear conditioning by baseline and inducible septo-hippocampal cyclin-dependent kinase 5. Neuropharmacology 44:1089-1099.

35. Sananbenesi F, et al. (2007) A hippocampal Cdk5 pathway regulates extinction of contextual fear. Nat Neurosci 10:1012-1019.

36. Abdipranoto A, Wu S, Stayte S, Vissel B (2008) The role of neurogenesis in neurodegenerative diseases and its implications for therapeutic development. CNS Neurol Disord Drug Targets 7:187-210.

37. Ko J, et al. (2001) p35 and p39 are essential for cyclin-dependent kinase 5 function during neurodevelopment. J Neurosci 21:6758-6771.

38. Yamaguchi M, Saito $H$, Suzuki M, Mori K (2000) Visualization of neurogenesis in the central nervous system using nestin promoter-GFP transgenic mice. Neuroreport 11:1991-1996.

39. Indra AK, et al. (1999) Temporally-controlled site-specific mutagenesis in the basal layer of the epidermis: Comparison of the recombinase activity of the tamoxifeninducible Cre-ER(T) and Cre-ER(T2) recombinases. Nucleic Acids Res 27:4324-4327.

40. Soriano P (1999) Generalized lacZ expression with the ROSA26 Cre reporter strain. Nat Genet 21:70-71.

41. Mandyam CD, Harburg GC, Eisch AJ (2007) Determination of key aspects of precursor cell proliferation, cell cycle length and kinetics in the adult mouse subgranular zone. Neuroscience 146:108-122. 